\title{
GREEN'S FUNCTION FOR A MULTIFIELD MATERIAL WITH A HEAT SOURCE
}

\author{
BOGDAN ROGOWSKI \\ Lodz University of Technology, Department of Mechanics of Materials, Eódź, Poland \\ e-mail: bogdan.rogowski@p.lodz.pl
}

Green's functions for a multifield material subjected to a point heat source are presented in an explicit analytical form. The study concerns the steady-state thermal loading infinite region, half-space region and two-constituent magneto-electro-thermo-elastic material region. The new mono-harmonic potential functions, obtained by the author, are used in the analysis. The elastic displacement, electric potential, magnetic potential and induced by those coupled multifield physical quantities, caused by internal or external heat sources, are limited and presented in a very useful form, exactly and explicitly.

Keywords: Green's functions, point heat source, multifield material, magneto-electro-thermo-elastic fundamental solution, multifield composites, exact solution

\section{Introduction}

The basic solutions, related among others to multifield materials, are Green's functions, which were first proposed by George Green in 1828. There are two different analysis processes for solutions in scientific literature. One has focused on the displacement, electric potential and magnetic potential, constructing equilibrium equations. The second has emphasized equilibrium equations of stresses, electric displacements and magnetic inductions as well as compatibility equations for strains. There is Stroh's formalism (Stroh, 1958) and Lekhnitskii's approach (Lekhnitskii, 1963), for example. On the other hand, there are three commonly used methods in analyzing boundary effects: the theoretical solution, numerical solution and the experiment. But, appropriate Green's functions for a thermoelastic half-space is a specific task. This is due to the fact that the fundamental solution for the displacements is not limited at infinity, which is inconsistent with the mechanical sense. For example, Hou et al. (2008) derived a solution with a logarithmic singularity in the generalized displacement fields. Thus, the consideration of static equilibrium of the thermoelastic half-space, a quarter of the space, an octant, a wedge, and a half-wedge under the action of a unit point (as well as distributed) of the internal heat source and boundary temperature or heat flux is a special and important task. The importance is dictated by the fact that the computational scheme of many structural elements is reduced to those volume material regions.

In the context of multifield materials, the solutions depend on a large number of material parameters. For magneto-electro-thermo-elastic materials, it is twenty one, making any solution other than explicit analytical one impractical. The exact formulae, in terms of elementary functions for multifield materials, are presented in this study. The generalized displacements have been obtained with an accuracy up to arbitrary constants, which do not affect the value of stresses. This is the major motivation of the study presented in this paper. Although it sounds theoretically more reasonable, experiment based verification is still desired. It is mentioned here that mono-harmonic potential functions can be found in Chen et al. (2004), but some simpler results, obtained by the author of this paper, are presented for the reader's convenience. 
The exact solutions related to crack and contact problems of multifield materials were recently presented by Rogowski (2012-2015) for instance.

\section{The thermoelastic fundamental solution for magneto-electro-thermo-elastic multifield materials}

\subsection{The fundamental equations for a magneto-electro-thermo-elastic medium}

We consider an axisymmetric problem. Assume that the field variables are functions of $r$ and $z$ in the cylindrical coordinate system $(r, \theta, z)$. Constitutive equations for a piezoelectric, piezomagnetic, electromagnetic and thermoelastic material polarized in the positive $z$-direction subjected to mechanical, thermal, magnetical and electrical fields can be written, in matrix representation, as

$$
\begin{aligned}
& \left\{\begin{array}{c}
\sigma_{r} \\
\sigma_{\theta} \\
\sigma_{z} \\
\sigma_{r z}
\end{array}\right\}=\left[\begin{array}{cccc}
c_{11} & c_{12} & c_{13} & 0 \\
c_{12} & c_{11} & c_{13} & 0 \\
c_{13} & c_{13} & c_{33} & 0 \\
0 & 0 & 0 & c_{44}
\end{array}\right]\left\{\begin{array}{l}
u_{r, r}-\alpha_{r} T \\
u_{r} / r-\alpha_{r} T \\
u_{z, z}-\alpha_{z} T \\
u_{r, z}+u_{z, r}
\end{array}\right\}+\left[\begin{array}{cc}
0 & e_{31} \\
0 & e_{31} \\
0 & e_{33} \\
e_{15} & 0
\end{array}\right]\left\{\begin{array}{l}
\phi_{, r} \\
\phi_{, z}
\end{array}\right\}+\left[\begin{array}{cc}
0 & q_{31} \\
0 & q_{31} \\
0 & q_{33} \\
q_{15} & 0
\end{array}\right]\left\{\begin{array}{l}
\psi_{, r} \\
\psi_{, z}
\end{array}\right\} \\
& \left\{\begin{array}{l}
D_{r} \\
D_{z}
\end{array}\right\}=\left[\begin{array}{cccc}
0 & 0 & 0 & e_{15} \\
e_{31} & e_{31} & e_{33} & 0
\end{array}\right]\left\{\begin{array}{l}
u_{r, r}+\alpha_{r} T \\
u_{r} / r+\alpha_{r} T \\
u_{z, z}+\alpha_{z} T \\
u_{r, z}+u_{z, r}
\end{array}\right\}-\left[\begin{array}{cc}
\varepsilon_{11} & 0 \\
0 & \varepsilon_{33}
\end{array}\right]\left\{\begin{array}{l}
\phi_{, r} \\
\phi_{, z}
\end{array}\right\}-\left[\begin{array}{cc}
d_{11} & 0 \\
0 & d_{33}
\end{array}\right]\left\{\begin{array}{l}
\psi_{, r} \\
\psi_{, z}
\end{array}\right\} \\
& \left\{\begin{array}{l}
B_{r} \\
B_{z}
\end{array}\right\}=\left[\begin{array}{cccc}
0 & 0 & 0 & q_{15} \\
q_{31} & q_{31} & q_{33} & 0
\end{array}\right]\left\{\begin{array}{l}
u_{r, r}+\alpha_{r} T \\
u_{r} / r+\alpha_{r} T \\
u_{z, z}+\alpha_{z} T \\
u_{r, z}+u_{z, r}
\end{array}\right\}-\left[\begin{array}{cc}
d_{11} & 0 \\
0 & d_{33}
\end{array}\right]\left\{\begin{array}{l}
\phi_{, r} \\
\phi_{, z}
\end{array}\right\}-\left[\begin{array}{cc}
\mu_{11} & 0 \\
0 & \mu_{33}
\end{array}\right]\left\{\begin{array}{l}
\psi_{, r} \\
\psi_{, z}
\end{array}\right\}
\end{aligned}
$$

where $\sigma_{i j}, D_{i}, B_{i}$ are mechanical stresses, electric displacements and magnetic inductions, respectively; $T$ is a temperature change; $c_{11}, c_{12}, c_{13}, c_{33}, c_{44}$ denote elastic stiffness; $\varepsilon_{11}, \varepsilon_{33}$, and $\mu_{11}, \mu_{33}$ denote dielectric permittivities and magnetic permeabilities, respectively; $e_{k l}, q_{k l}$ and $d_{l l}$ are piezoelectric, piezomagnetic and magnetoelectric coefficients, respectively, and $u_{r}, u_{z}$ are mechanical displacements, while $\phi$ and $\psi$ are electric and magnetic potentials, respectively; $\alpha_{r}$ and $\alpha_{z}$ are thermal expansion coefficients. The subscripts following a comma denote partial differentation with respect to the indicated variables. We mention that various uncoupled cases can be reduced by setting the appropriate coupling coefficients to zero.

The equilibrium equations and the Maxwell equations, in the absence of body forces, electric and magnetic charge densities are given by

$$
\begin{array}{ll}
\frac{\partial \sigma_{r}}{\partial r}+\frac{\partial \sigma_{r z}}{\partial z}+\frac{\sigma_{r}-\sigma_{\theta}}{r}=0 & \frac{\partial \sigma_{r z}}{\partial r}+\frac{\partial \sigma_{z}}{\partial z}+\frac{\sigma_{r z}}{r}=0 \\
\frac{\partial D_{r}}{\partial r}+\frac{\partial D_{z}}{\partial z}+\frac{D_{r}}{r}=0 & \frac{\partial B_{r}}{\partial r}+\frac{\partial B_{z}}{\partial z}+\frac{B_{r}}{r}=0
\end{array}
$$

The temperature field in the medium without heat generation in a steady-state is governed by the following equation

$$
\lambda_{r}\left(\frac{\partial^{2} T}{\partial r^{2}}+\frac{1}{r} \frac{\partial T}{\partial r}\right)+\lambda_{z} \frac{\partial^{2} T}{\partial z^{2}}=0
$$

where $\lambda_{r}, \lambda_{z}$ are coefficients of thermal conductivity. Substituting constitutive equations (2.1) into equilibrium equations (2.2) yields the basic governing equilibrium equations for the displa- 
cements $u_{r}$ and $u_{z}$, electric potential $\phi$ and magnetic potential $\psi$ as follows

$$
\begin{aligned}
& c_{11} B_{1} u_{r}+c_{44} D^{2} u_{r}+\left(c_{13}+c_{44}\right) D \frac{\partial u_{z}}{\partial r}+\left(e_{15}+e_{31}\right) D \frac{\partial \phi}{\partial r}+\left(q_{15}+q_{31}\right) D \frac{\partial \psi}{\partial r}-\beta_{1} \frac{\partial T}{\partial r}=0 \\
& c_{44} B_{0} u_{z}+c_{33} D^{2} u_{z}+\left(c_{13}+c_{44}\right) D \frac{\partial\left[r u_{r}\right]}{r \partial r}+\left(e_{15} B_{0}+e_{33} D^{2}\right) \phi \\
& \quad+\left(q_{15} B_{0}+q_{33} D^{2}\right) \psi-\beta_{3} D T=0 \\
& \left(e_{15}+e_{31}\right) D \frac{\partial\left[r u_{r}\right]}{r \partial r}+\left(e_{15} B_{0}+e_{33} D^{2}\right) u_{z}-\left(\varepsilon_{11} B_{0}+e_{33} D^{2}\right) \phi \\
& \quad-\left(d_{11} B_{0}+d_{33} D^{2}\right) \psi+p_{3} D T=0 \\
& \left(q_{15}+q_{31}\right) D \frac{\partial\left[r u_{r}\right]}{r \partial r}+\left(q_{15} B_{0}+q_{33} D^{2}\right) u_{z}-\left(d_{11} B_{0}+d_{33} D^{2}\right) \phi \\
& \quad-\left(\mu_{11} B_{0}+\mu_{33} D^{2}\right) \psi+\gamma_{3} D T=0
\end{aligned}
$$

where the following differential operators have been introduced

$$
B_{k}=\frac{\partial^{2}}{\partial r^{2}}+\frac{1}{r} \frac{\partial}{\partial r}-\frac{k}{r^{2}} \quad k=0,1 \quad D=\frac{\partial}{\partial z} \quad D^{2}=\frac{\partial^{2}}{\partial z^{2}}
$$

In addition, $\beta_{i}$ are the thermal moduli and $p_{3}, \gamma_{3}$ are pyroelectric and pyromagnetic constants, respectively, defined by

$$
\begin{array}{ll}
\beta_{1}=\left(c_{11}+c_{12}\right) \alpha_{r}+c_{13} \alpha_{z} & \beta_{3}=2 c_{13} \alpha_{r}+c_{33} \alpha_{z} \\
p_{3}=2 e_{31} \alpha_{r}+e_{33} \alpha_{z} & \gamma_{3}=2 q_{31} \alpha_{r}+q_{33} \alpha_{z}
\end{array}
$$

Equations (2.1) to (2.3) contain 13 equations and 13 unknowns. The 13 unknowns are: two elastic displacements, fourth stresses, two electric displacements and two magnetic inductions, one electric and one magnetic potential and temperature change of the body. Therefore, the 13 unknowns can be determined by solving the 13 equations (2.1) to (2.3).

The governing equations are generalized equilibrium equations (2.4) and heat conduction equation (2.3), which induces five unknowns. These are: two displacements, one electric and one magnetic potential and temperature change of the body.

The transversely isotropic multifield material is characterized by 17 material constants. If the effect of temperature change is taken into account then also four thermal constants appear in the analysis.

Based on the method named the Schmidt method (Morse and Feshbach, 1953) the general solution to the governing equations are obtained by the generalized Almansi theorem.

Then equations (2.4) can be further simplified to

$$
\begin{array}{ll}
u_{r}(r, z)=\sum_{i=0}^{4} \alpha_{1 i} \lambda_{i} \frac{\partial \varphi_{i}}{\partial r} & u_{z}(r, z)=\sum_{i=0}^{4} \frac{1}{\lambda_{i}} \frac{\partial \varphi_{i}}{\partial z} \\
\phi(r, z)=-\sum_{i=0}^{4} \frac{\alpha_{3 i}}{\lambda_{i}} \frac{\partial \varphi_{i}}{\partial z} & \psi(r, z)=-\sum_{i=0}^{4} \frac{\alpha_{4 i}}{\lambda_{i}} \frac{\partial \varphi_{i}}{\partial z} \\
T(r, z)=\frac{\alpha_{00}}{\lambda_{0}^{2}} \frac{\partial^{2} \varphi_{0}}{\partial z^{2}} &
\end{array}
$$

where

$$
\begin{aligned}
a_{1 i} & =\frac{a_{1} \lambda_{i}^{6}+b_{1} \lambda_{i}^{4}+c_{1} \lambda_{i}^{2}+d_{1}}{a_{2} \lambda_{i}^{6}+b_{2} \lambda_{i}^{4}+c_{2} \lambda_{i}^{2}+d_{2}} \frac{1}{\lambda_{i}^{2}} & a_{3 i} & =\frac{a_{3} \lambda_{i}^{6}+b_{3} \lambda_{i}^{4}+c_{3} \lambda_{i}^{2}+d_{3}}{a_{2} \lambda_{i}^{6}+b_{2} \lambda_{i}^{4}+c_{2} \lambda_{i}^{2}+d_{2}} \\
a_{4 i} & =\frac{a_{4} \lambda_{i}^{6}+b_{4} \lambda_{i}^{4}+c_{4} \lambda_{i}^{2}+d_{4}}{a_{2} \lambda_{i}^{6}+b_{2} \lambda_{i}^{4}+c_{2} \lambda_{i}^{2}+d_{2}} & a_{00} & =\frac{a \lambda_{0}^{8}+b \lambda_{0}^{6}+c \lambda_{0}^{4}+d \lambda_{0}^{2}+e}{a_{2} \lambda_{0}^{6}+b_{2} \lambda_{0}^{4}+c_{2} \lambda_{0}^{2}+d_{2}}
\end{aligned}
$$


where $\lambda_{0}^{2}=\lambda_{r} / \lambda_{z}$ and $\lambda_{i}^{2}$ are the roots of the following characteristic algebraic equation

$$
a \lambda^{8}+b \lambda^{6}+c \lambda^{4}+d \lambda^{2}+e=0
$$

whose parameters $a, b, c, d$ and $e$ and roots (eigenvalues) $\lambda_{i}^{2}(i=1,2,3,4)$ are given in Appen$\operatorname{dix}$ A.

The mono-harmonic functions satisfy the equations

$$
\left(\Delta+\frac{1}{\lambda_{i}^{2}} \frac{\partial^{2}}{\partial z^{2}}\right) \varphi_{i}(r, z)=0 \quad i=0,1,2,3,4
$$

The parameters $a_{1}, b_{1}, c_{1}, d_{1}, b_{2}, c_{2}, d_{2}$ and coefficients $a_{3 i}$ and $a_{4 i}$, which are defined by the coefficient $a_{1 i}$, are listed in Appendix A for the reader's convenience.

The stresses are

$$
\begin{aligned}
\sigma_{r} & =-\sum_{i=0}^{4} \frac{\alpha_{5 i}}{\lambda_{i}} \frac{\partial^{2} \varphi_{i}}{\partial z^{2}}-\left(c_{11}-c_{12}\right) \frac{u_{r}}{r}-\beta_{1} T \\
\sigma_{\theta} & =-\sum_{i=0}^{4} \frac{\alpha_{5 i}}{\lambda_{i}} \frac{\partial^{2} \varphi_{i}}{\partial z^{2}}-\left(c_{11}-c_{12}\right) \frac{\partial u_{r}}{\partial r}-\beta_{1} T \\
\sigma_{z} & =\sum_{i=0}^{4} \frac{\alpha_{5 i}}{\lambda_{i}^{3}} \frac{\partial^{2} \varphi_{i}}{\partial z^{2}}+\lambda_{0}^{-2} \beta_{1} T \quad \sigma_{z r}=\sum_{i=0}^{4} \frac{\alpha_{5 i}}{\lambda_{i}} \frac{\partial^{2} \varphi_{i}}{\partial r \partial z}+\lambda_{0}^{-2} \beta_{1} a_{00} \frac{\partial^{2} \varphi_{0}}{\partial r \partial z}
\end{aligned}
$$

where

$$
a_{5 i}=c_{11} a_{1 i}-c_{13}+e_{31} a_{3 i}+q_{31} a_{4 i}
$$

The components of the electric field vector $E_{r}$ and $E_{z}$ are

$$
E_{r}=-\frac{\partial \phi}{\partial r}=\sum_{i=0}^{4} \frac{\alpha_{3 i}}{\lambda_{i}} \frac{\partial^{2} \varphi_{i}}{\partial r \partial z} \quad E_{z}=-\frac{\partial \phi}{\partial z}=\sum_{i=0}^{4} \frac{\alpha_{3 i}}{\lambda_{i}} \frac{\partial^{2} \varphi_{i}}{\partial z^{2}}
$$

The electric displacements are

$$
\begin{aligned}
& D_{r}=e_{15}\left(\frac{\partial u_{r}}{\partial z}+\frac{\partial u_{z}}{\partial r}\right)+\varepsilon_{11} E_{r}+d_{11} H_{r}=\sum_{i=0}^{4} a_{6 i} \lambda_{i} \frac{\partial^{2} \varphi_{i}}{\partial r \partial z} \\
& D_{z}=e_{31}\left(\frac{\partial u_{r}}{\partial r}+\frac{u_{r}}{r}\right)+e_{33} \frac{\partial u_{z}}{\partial z}+\varepsilon_{33} E_{z}+d_{33} H_{z}+\beta_{3} T=\sum_{i=0}^{4} \frac{a_{6 i}}{\lambda_{i}} \frac{\partial^{2} \varphi_{i}}{\partial z^{2}}
\end{aligned}
$$

where

$$
a_{6 i}=e_{15} a_{1 i}+\frac{e_{15}+\varepsilon_{11} a_{3 i}+d_{11} a_{4 i}}{\lambda_{i}^{2}}
$$

The components of the magnetic field vector $H_{r}$ and $H_{z}$ are

$$
H_{r}=-\frac{\partial \psi}{\partial r}=\sum_{i=0}^{4} \frac{\alpha_{4 i}}{\lambda_{i}} \frac{\partial^{2} \varphi_{i}}{\partial r \partial z} \quad H_{z}=-\frac{\partial \psi}{\partial z}=\sum_{i=0}^{4} \frac{\alpha_{4 i}}{\lambda_{i}} \frac{\partial^{2} \varphi_{i}}{\partial z^{2}}
$$

The magnetic inductions are

$$
\begin{aligned}
& B_{r}=q_{15}\left(\frac{\partial u_{r}}{\partial z}+\frac{\partial u_{z}}{\partial r}\right)+\mu_{11} H_{r}+d_{11} E_{r}=\sum_{i=0}^{4} a_{7 i} \lambda_{i} \frac{\partial^{2} \varphi_{i}}{\partial r \partial z} \\
& B_{z}=q_{31}\left(\frac{\partial u_{r}}{\partial r}+\frac{u_{r}}{r}\right)+q_{33} \frac{\partial u_{z}}{\partial z}+\mu_{33} H_{z}+d_{33} E_{z}+\gamma_{3} T=\sum_{i=0}^{4} \frac{a_{7 i}}{\lambda_{i}} \frac{\partial^{2} \varphi_{i}}{\partial z^{2}}
\end{aligned}
$$

where

$$
a_{7 i}=q_{15} a_{1 i}+\frac{q_{15}+\mu_{11} a_{4 i}+d_{11} a_{3 i}}{\lambda_{i}^{2}}
$$




\section{Thermal problems for multifield materials}

Consider the problem of a point heat source placed within the multifield material. Introduce the following mono-harmonic functions, which are even functions with respect to the $z$-coordinate

$$
\begin{aligned}
& \varphi_{i}\left(r, z_{i}\right)=A_{i}\left[z_{i} \arcsin h\left(\frac{z_{i}}{r}\right)-R_{i}\right] \quad z_{i}=\lambda_{i} z \quad R_{i}=\sqrt{r^{2}+z_{i}^{2}} \\
& i=0,1,2,3,4
\end{aligned}
$$

where $A_{i}$ are constants to be determined.

The derivatives $\varphi_{i}$ are as follows

$$
\begin{array}{llll}
\frac{\partial \varphi_{i}}{\partial r}=-\frac{R_{i}}{r} & \frac{\partial \varphi_{i}}{\partial z_{i}}=\arcsin h\left(\frac{z_{i}}{r}\right) & \frac{\partial^{2} \varphi_{i}}{\partial r \partial z_{i}}=-\frac{z_{i}}{r R_{i}} \\
\frac{\partial^{2} \varphi_{i}}{\partial z_{i}^{2}}=\frac{1}{R_{i}} & \frac{\partial^{2} \varphi_{i}}{\partial r^{2}}=\frac{z_{i}^{2}}{r^{2} R_{i}} & \frac{\partial^{2} \varphi_{i}}{\partial r^{2}}+\frac{1}{r} \frac{\partial \varphi_{i}}{\partial r}+\frac{\partial^{2} \varphi_{i}}{\partial z_{i}^{2}}=0
\end{array}
$$

The physical multifields are as follows

$$
\begin{array}{rlrl}
u_{r} & =-\sum A_{i} a_{1 i} \lambda_{i} \frac{R_{i}}{r} \quad & \left(u_{z}, \phi, \psi\right) & =\sum A_{i}\left(1,-a_{3 i},-a_{4 i}\right) \arcsin h\left(\frac{z_{i}}{r}\right) \\
T & =A_{0} a_{00} \frac{1}{R_{0}} & \frac{\partial T}{\partial z}=-A_{0} a_{00} \frac{\lambda_{0}^{2} z}{R_{0}^{3}} & \frac{\partial T}{\partial r}=-A_{0} a_{00} \frac{r}{R_{0}^{3}} \\
\sigma_{r} & =-\sum A_{i} a_{5 i} \lambda_{i} \frac{1}{R_{i}}+\left(c_{11}-c_{12}\right) \sum A_{i} a_{1 i} \lambda_{i} \frac{R_{i}}{r^{2}}-\beta_{1} T \\
\sigma_{\theta} & =-\sum A_{i} a_{5 i} \lambda_{i} \frac{1}{R_{i}}-\left(c_{11}-c_{12}\right) \sum A_{i} a_{1 i} \lambda_{i}\left(\frac{R_{i}}{r^{2}}-\frac{1}{R_{i}}\right)-\beta_{1} T \\
\sigma_{z} & =\sum A_{i} \frac{a_{5 i}}{\lambda_{i}} \frac{1}{R_{i}}+\frac{\beta_{1}}{\lambda_{0}^{2}} T & \sigma_{z r} & =-\sum A_{i} a_{5 i} \frac{z_{i}}{r R_{i}}-\frac{\beta_{1}}{\lambda_{0}} \frac{z}{r} T \\
E_{r} & =-\sum A_{i} a_{3 i} \frac{z_{i}}{r R_{i}} & E_{z} & =\sum A_{i} a_{3 i} \lambda_{i} \frac{1}{R_{i}} \\
H_{r} & =-\sum A_{i} a_{4 i} \frac{z_{i}}{r R_{i}} & H_{z} & =\sum A_{i} a_{4 i} \lambda_{i} \frac{1}{R_{i}} \\
D_{r} & =\sum A_{i} a_{6 i} \lambda_{i}^{2} \frac{z_{i}}{r R_{i}} & D_{z} & =\sum A_{i} a_{6 i} \lambda_{i} \frac{1}{R_{i}} \\
B_{r} & =\sum A_{i} a_{7 i} \lambda_{i}^{2} \frac{z_{i}}{r R_{i}} & B_{z} & =\sum A_{i} a_{7 i} \lambda_{i} \frac{1}{R_{i}}
\end{array}
$$

where the following abbreviation notation is used $\sum=\sum_{i=0}^{4}$.

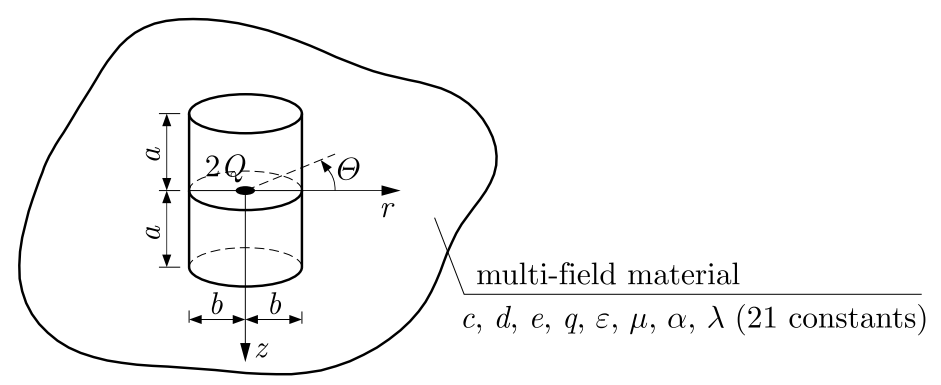

Fig. 1. A point heat source $2 Q$ in an infinite multifield material

When we use the physical consideration that the total heat flux transmitted through a cylinder $0 \leqslant z \leqslant a, r \leqslant b$ must be equal to a point heat source $Q$ (see Fig. 1), the following equation can be written 


$$
-2 \pi \lambda_{z} \int_{0}^{b} \frac{\partial T}{\partial z}(r, a) r d r-2 \pi \lambda_{r} \int_{0}^{a} \frac{\partial T}{\partial r}(b, z) d z=Q
$$

The substitution of $\mathrm{Eq}(3.3)_{4,5}$ and integrations yields

$$
\begin{aligned}
\frac{Q}{2 \pi} & =\lambda_{z} \lambda_{0}^{2} a a_{00} A_{0} \int_{0}^{b} \frac{r}{\sqrt{\left(r^{2}+\lambda_{0}^{2} a^{2}\right)^{3}}} d r+\lambda_{r} b^{2} a_{00} A_{0} \int_{0}^{a} \frac{1}{\sqrt{\left(b^{2}+\lambda_{0}^{2} z^{2}\right)^{3}}} d z \\
& =\left.\lambda_{r} a a_{00} A_{0}\left(-\frac{1}{\sqrt{r^{2}+\lambda_{0}^{2} a^{2}}}\right)\right|_{0} ^{b}-\left.\lambda_{r} a_{00} A_{0}\left(1-\frac{z}{\sqrt{b^{2}+\lambda_{0}^{2} z^{2}}}\right)\right|_{0} ^{a} \\
& =\lambda_{r} a_{00} A_{0}\left(-\frac{a}{\sqrt{b^{2}+\lambda_{0}^{2} a^{2}}}+\frac{1}{\lambda_{0}}+\frac{a}{\sqrt{b^{2}+\lambda_{0}^{2} a^{2}}}\right)=a_{00} A_{0} \sqrt{\lambda_{r} \lambda_{z}}
\end{aligned}
$$

that is

$$
A_{0}=\frac{Q}{2 \pi a_{00} \sqrt{\lambda_{r} \lambda_{z}}}
$$

Note that in an infinite medium with the point heat source $2 Q$, the constant $A_{0}$ assumes the same value. The temperature and heat fluxes are

$$
T=\frac{Q}{2 \pi \sqrt{\lambda_{r} \lambda_{z}}} \frac{1}{R_{0}} \quad \lambda_{z} \frac{\partial T}{\partial z}=-\frac{Q}{2 \pi} \frac{\lambda_{0} z}{R_{0}^{3}} \quad \lambda_{r} \frac{\partial T}{\partial r}=-\frac{Q}{2 \pi} \frac{\lambda_{0} r}{R_{0}^{3}}
$$

Of course

$$
\lambda_{r}\left(\frac{\partial^{2} T}{\partial r^{2}}+\frac{1}{r} \frac{\partial T}{\partial r}\right)+\lambda_{z} \frac{\partial^{2} T}{\partial z^{2}}=0
$$

\subsection{The half-space problem}

The boundary conditions and the corresponding equations for $A_{i}$ are

(a) $\quad \sigma_{z r}(r, 0)=0 \quad$ is identically satisfied

(b) $\quad \sigma_{z}(r, 0)=0 \quad \sum_{i=1}^{4} A_{i} \frac{a_{5 i}}{\lambda_{i}}+\frac{Q}{2 \pi \lambda_{z} a_{00}}\left(\frac{\beta_{1} a_{00}}{\lambda_{0}}+a_{50}\right) \frac{1}{\lambda_{0}^{2}}=0$

(c) $\quad D_{z}(r, 0)=0 \quad \sum_{i=1}^{4} A_{i} a_{6 i} \lambda_{i}+\frac{Q}{2 \pi \lambda_{z} a_{00}} a_{60}=0$

(d) $\quad B_{z}(r, 0)=0 \quad \sum_{i=1}^{4} A_{i} a_{7 i} \lambda_{i}+\frac{Q}{2 \pi \lambda_{z} a_{00}} a_{70}=0$

(e) $u_{r}$ is finite at $r=0 \quad \sum_{i=1}^{4} A_{i} a_{1 i} \lambda_{i}^{2}+\frac{Q}{2 \pi \lambda_{z} a_{00}} a_{10} \lambda_{0}=0$

Thus, the coupled field in a semi-infinite transversely isotropic multifield material is determined by solution (3.3) and the following constants $A_{i} \lambda_{i}$

$$
\left[\begin{array}{l}
A_{1} \lambda_{1} \\
A_{2} \lambda_{2} \\
A_{3} \lambda_{3} \\
A_{4} \lambda_{4}
\end{array}\right]=-\frac{Q}{2 \pi \lambda_{z} a_{00}}\left[\begin{array}{cccc}
\frac{a_{51}}{\lambda_{1}^{2}} & \frac{a_{52}}{\lambda_{2}^{2}} & \frac{a_{53}}{\lambda_{3}^{2}} & \frac{a_{54}}{\lambda_{4}^{2}} \\
a_{61} & a_{62} & a_{63} & a_{64} \\
a_{71} & a_{72} & a_{73} & a_{74} \\
a_{11} \lambda_{1} & a_{12} \lambda_{2} & a_{13} \lambda_{3} & a_{14} \lambda_{4}
\end{array}\right]^{-1}\left[\begin{array}{c}
\frac{1}{\lambda_{0}^{2}}\left(\frac{\beta_{1} a_{00}}{\lambda_{0}}+a_{50}\right) \\
a_{60} \\
a_{70} \\
a_{10} \lambda_{0}
\end{array}\right]
$$


Generally, the permittivity and permeability of air or vacuum is about 680 and 475 times smaller, respectively, than that of commercial multifield materials. In reality, $D_{z}$ and $B_{z}$ do not transmit through the free boundary of half-space as assumed in conditions (c) and (d) of equations (3.9). It can be seen from equations (3.10) and (3.3) that Green's functions for point heat sources applied on the boundary of the half-space are expressed exactly and explicitly in terms of elementary functions. This will be greatly beneficial to the succeeding analysis of thermoelastic problems of magneto-electro-thermo-elastic materials. Note that the total heat flux transmitted through the free boundary $z=0$ is

$$
Q+2 \pi \lambda_{z} \int_{0}^{\infty} \frac{\partial T}{\partial z} r d r=Q+\left.Q \frac{\lambda_{0} z}{R_{0}}\right|_{0} ^{\infty}=Q-Q=0
$$

This is a confirmation of the correctness of the obtained result. Note again that

$$
\begin{array}{ll}
a_{5 i}=c_{11} a_{1 i}-c_{13}+e_{31} a_{3 i}+q_{31} a_{4 i} & a_{6 i}=e_{15} a_{1 i}+\frac{e_{15}+\varepsilon_{11} a_{3 i}+d_{11} a_{4 i}}{\lambda_{i}^{2}} \\
a_{7 i}=q_{15} a_{1 i}+\frac{q_{15}+\mu_{11} a_{4 i}+d_{11} a_{3 i}}{\lambda_{i}^{2}} &
\end{array}
$$

and $a_{1 i}, a_{3 i}, a_{4 i}$ are defined by equations (3.15), see also very coupled but alternative equations (A2) and (A3) in Appendix A and equations (3.22) in special cases.

\subsection{An infinite body containing a point heat source $2 Q$}

If

$$
\sum_{i=0}^{4} A_{i} a_{1 i} \lambda_{i}^{2}=0 \quad \text { and } \quad \sum_{i=0}^{4} A_{i}\left(1,-a_{3 i},-a_{4 i}\right)=0
$$

then the generalized displacements $u_{r}, u_{z}, \phi$ and $\psi$ caused by the internal heat source $2 Q$ are limited, but they cannot be calculated in the neighborhood of the $z$-axis, that is when $r \rightarrow 0$. The displacements which are obtained with an accuracy up to an arbitrary constant do not affect the value of stresses. Arbitrary constants can be treated as linear displacements of the medium as a rigid body in the axial direction without rotation.

The solution to algebraic system of equations (3.13) is

$$
\left[\begin{array}{c}
A_{1} \\
A_{2} \\
A_{3} \\
A_{4}
\end{array}\right]=-\frac{Q}{2 \pi a_{00} \sqrt{\lambda_{r} \lambda_{z}}}\left[\begin{array}{cccc}
1 & 1 & 1 & 1 \\
a_{31} & a_{32} & a_{33} & a_{34} \\
a_{41} & a_{42} & a_{43} & a_{44} \\
a_{11} \lambda_{1}^{2} & a_{12} \lambda_{2}^{2} & a_{13} \lambda_{3}^{2} & a_{14} \lambda_{4}^{2}
\end{array}\right]^{-1}\left[\begin{array}{c}
1 \\
a_{30} \\
a_{40} \\
a_{10} \lambda_{0}^{2}
\end{array}\right]
$$

where the coefficients $a_{1 i}, a_{3 i}, a_{4 i}$ for $i=0,1,2,3,4$ are as follows

$$
\left\{\begin{array}{l}
a_{1 i} \\
a_{3 i} \\
a_{4 i}
\end{array}\right\}=\left[\begin{array}{ccc}
\left(e_{31}+e_{15}\right) \lambda_{i}^{2} & \varepsilon_{11}-\varepsilon_{33} \lambda_{i}^{2} & d_{11}-d_{33} \lambda_{i}^{2} \\
\left(q_{31}+q_{15}\right) \lambda_{i}^{2} & d_{11}-d_{33} \lambda_{i}^{2} & \mu_{11}-\mu_{33} \lambda_{i}^{2} \\
c_{11}+c_{13} \lambda_{i}^{2} & e_{31}+e_{33} \lambda_{i}^{2} & q_{31}+q_{33} \lambda_{i}^{2}
\end{array}\right]^{-1}\left\{\begin{array}{c}
e_{33} \lambda_{i}^{2}-e_{15}+p_{3} a_{00} \lambda_{i} \delta_{i 0} \\
q_{33} \lambda_{i}^{2}-q_{15}+\gamma_{3} a_{00} \lambda_{i} \delta_{i 0} \\
c_{33} \lambda_{i}^{2}+c_{13}-\left(\beta_{3}+\beta_{1} \lambda_{i}^{-2}\right) a_{00} \lambda_{i} \delta_{i 0}
\end{array}\right\}
$$

This is an alternative and simpler form of parameters defined by equations (A2) and (A3).

Note that the units of the elements of the last matrix are for typical multifield materials

$$
\begin{array}{lrrr}
{[e]=\mathrm{C} / \mathrm{m}^{2}} & {\left[p_{3}\right]=10^{-6} \mathrm{C} /\left(\mathrm{m}^{2} \mathrm{~K}\right)} & {\left[a_{00}\right]=10^{6} \mathrm{~K}} & {\left[p_{3} a_{00}\right]=\mathrm{C} / \mathrm{m}^{2}} \\
{[q]=10^{2} \mathrm{~N} /(\mathrm{Am})} & {\left[\gamma_{3}\right]=10^{-4} \mathrm{~N} /(\mathrm{AmK})} & {\left[\gamma_{3} a_{00}\right]=10^{2} \mathrm{~N} /(\mathrm{Am})} \\
{[c]=10^{10} \mathrm{~N} / \mathrm{m}^{2}} & {\left[\beta_{1}, \beta_{3}\right]=10^{4} \mathrm{~N} /\left(\mathrm{m}^{2} \mathrm{~K}\right)} & {\left[\left(\beta_{1}, \beta_{3}\right) a_{00}\right]=10^{10} \mathrm{~N} / \mathrm{m}^{2}}
\end{array}
$$

with the multiplier $\in\langle 1,10\rangle$. 
This states that the constituents of the sums are of the same order in each row of the last matrix in (3.15).

Green's functions for the internal heat source applied in multifield materials are determined by equations (3.3), (3.14) and (3.15). All physical components of multifield materials are expressed in forms of elementary functions. It is very simple and straightforward to give numerical results. The results may help the understanding of behaviour of "smart" devices and "intelligent" structures made by multifield materials.

\subsection{Single phase materials and multifield composite materials}

Multifield composite materials usually comprise alternating piezoelectric and piezomagnetic materials. If the material is piezoelectric then we define the matrix

$$
\mathbf{C}_{E}=\left[\begin{array}{ccc}
\left(e_{31}+e_{15}\right) \lambda_{i}^{2} & \varepsilon_{11}-\varepsilon_{33} \lambda_{i}^{2} & 0 \\
0 & 0 & -\infty \\
c_{11}+c_{13} \lambda_{i}^{2} & e_{31}+e_{33} \lambda_{i}^{2} & 0
\end{array}\right]
$$

and its inverse matrix

$$
\begin{aligned}
& \mathbf{C}_{E}^{-1}=\frac{1}{\Delta_{E}}\left[\begin{array}{ccc}
e_{31}+e_{33} \lambda_{i}^{2} & 0 & -\left(\varepsilon_{11}-\varepsilon_{33} \lambda_{i}^{2}\right) \\
-\left(c_{11}+c_{13} \lambda_{i}^{2}\right) & 0 & \left(e_{31}+e_{15}\right) \lambda_{i}^{2} \\
0 & 0 & 0
\end{array}\right] \\
& \Delta_{E}=\left(e_{31}+e_{15}\right) \lambda_{i}^{2}\left(e_{31}+e_{33} \lambda_{i}^{2}\right)-\left(c_{11}+c_{13} \lambda_{i}^{2}\right)\left(\varepsilon_{11}-\varepsilon_{33} \lambda_{i}^{2}\right)
\end{aligned}
$$

Of course

$$
\mathbf{C}_{E} \mathbf{C}_{E}^{-1}=\left[\begin{array}{lll}
1 & 0 & 0 \\
0 & 1 & 0 \\
0 & 0 & 1
\end{array}\right]
$$

For piezomagnetic material, it is

$$
\mathbf{C}_{H}=\left[\begin{array}{ccc}
0 & -\infty & 0 \\
\left(q_{31}+q_{15}\right) \lambda_{i}^{2} & 0 & \mu_{11}-\mu_{33} \lambda_{i}^{2} \\
c_{11}+c_{13} \lambda_{i}^{2} & 0 & q_{31}+q_{33} \lambda_{i}^{2}
\end{array}\right]
$$

Then we obtain

$$
\begin{aligned}
& \mathbf{C}_{H}^{-1}=\frac{1}{\Delta_{H}}\left[\begin{array}{ccc}
0 & q_{31}+q_{33} \lambda_{i}^{2} & -\left(\mu_{11}-\mu_{33} \lambda_{i}^{2}\right) \\
0 & 0 & 0 \\
0 & -\left(c_{11}+c_{13} \lambda_{i}^{2}\right) & \left(q_{31}+q_{15}\right) \lambda_{i}^{2}
\end{array}\right] \\
& \Delta_{H}=\left(q_{31}+q_{15}\right) \lambda_{i}^{2}\left(q_{31}+q_{33} \lambda_{i}^{2}\right)-\left(c_{11}+c_{13} \lambda_{i}^{2}\right)\left(\mu_{11}-\mu_{33} \lambda_{i}^{2}\right)
\end{aligned}
$$

where $\mathbf{C}_{H} \mathbf{C}_{H}^{-1}=\mathbf{I}$; $\mathbf{I}$ is the square unit matrix.

Thus

$$
\begin{aligned}
& \left\{\begin{array}{l}
a_{1 i} \\
a_{3 i}
\end{array}\right\}^{E}=\frac{1}{\Delta_{E}}\left[\begin{array}{cc}
e_{31}+e_{33} \lambda_{i}^{2} & -\left(\varepsilon_{11}-\varepsilon_{33} \lambda_{i}^{2}\right) \\
-\left(c_{11}+c_{13} \lambda_{i}^{2}\right) & \left(e_{31}+e_{15}\right) \lambda_{i}^{2}
\end{array}\right]\left\{\begin{array}{c}
e_{33} \lambda_{i}^{2}-e_{15}+p_{3} a_{00} \lambda_{i} \delta_{i 0} \\
\left.c_{33} \lambda_{i}^{2}+c_{13}-\left(\beta_{3}+\beta_{1} \lambda_{i}^{-2}\right) a_{00} \lambda_{i} \delta_{i 0}\right\}
\end{array}\right. \\
& \left\{\begin{array}{l}
a_{1 i} \\
a_{4 i}
\end{array}\right\}^{H}=\frac{1}{\Delta_{H}}\left[\begin{array}{cc}
q_{31}+q_{33} \lambda_{i}^{2} & -\left(\mu_{11}-\mu_{33} \lambda_{i}^{2}\right) \\
-\left(c_{11}+c_{13} \lambda_{i}^{2}\right) & \left(q_{31}+q_{15}\right) \lambda_{i}^{2}
\end{array}\right]\left\{\begin{array}{c}
q_{33} \lambda_{i}^{2}-q_{15}+\gamma_{3} a_{00} \lambda_{i} \delta_{i 0} \\
c_{33} \lambda_{i}^{2}+c_{13}-\left(\beta_{3}+\beta_{1} \lambda_{i}^{-2}\right) a_{00} \lambda_{i} \delta_{i 0}
\end{array}\right\}
\end{aligned}
$$

respectively for the piezoelectric and piezomagnetic thermoelastic materials. 
Note that for the piezoelectric material is $a_{4 i}=0$, but $a_{3 i}$ defines $a_{7 i}$, that is also $B_{z}$ by the electromagnetic constant $d_{11}$. Similarly is for the piezomagnetic material where $a_{3 i}=0$, but $a_{4 i}$ defines $a_{6 i}$, that is also $D_{z}$ as a consequence of the electromagnetic effect (see equations (3.12)).

Fore the two-phase multifield material, the constant $A_{0}$ will be

$$
A_{0}=\frac{Q}{\pi\left(a_{00} \sqrt{\lambda_{r} \lambda_{z}}+a_{00}^{\prime} \sqrt{\lambda_{r}^{\prime} \lambda_{z}^{\prime}}\right)}
$$

where the material parameter of the second material is denoted by prime.

The inverse matrices are obtained as arithmetically average values in this case. Since the plane $z=0$ is a plane of symmetry $\left(\sigma_{z r}=0, H_{r}=0, E_{r}=0, u_{z}=0, \phi=0\right.$ and $\psi=0$ on this plane), the solutions may be used for the two-phase multifield composite material.

\subsection{Solution for a purely thermoelastic material}

For a transversely isotropic thermoelastic medium, the temperature field is the same as that obtained in Section 3 and described by equations (3.7). The thermoelastic solution for the purely elastic problem can be easily derived from that of the piezoelectric material (on assumption that $\varepsilon_{11}-\varepsilon_{33} \lambda_{i}^{2} \rightarrow \infty$ and $e_{31}=e_{33}=e_{15}=0$ ) or the piezomagnetic material (by assuming $\mu_{11}-\mu_{33} \lambda_{i}^{2} \rightarrow \infty$ and $\left.q_{31}=q_{33}=q_{15}=0\right)$. Both formulae (3.22) give the same result

$$
a_{1 i}=\frac{c_{33} \lambda_{i}^{2}+c_{13}-\left(\beta_{3}+\beta_{1} \lambda_{i}^{-2}\right) a_{00} \lambda_{i} \delta_{i 0}}{c_{11}+c_{13} \lambda_{i}^{2}} \quad i=0,1,2
$$

and equations (2.12) and (2.8) yield

$$
a_{5 i}=c_{11} a_{1 i}-c_{13} \quad a_{00}=\frac{c_{44} c_{33}\left(\lambda_{0}^{2}-\lambda_{1}^{2}\right)\left(\lambda_{0}^{2}-\lambda_{2}^{2}\right)}{\beta_{1}\left(c_{33} \lambda_{0}^{2}-c_{44}\right)-\beta_{3} \lambda_{0}^{2}\left(c_{13}+c_{44}\right)}
$$

The remaining material parameters $a_{3 i}, a_{4 i}, a_{6 i}$ and $a_{7 i}$ vanish.

The constants $A_{1}$ and $A_{2}$ are obtained as follows

$$
\begin{aligned}
& A_{1}=-\frac{Q \lambda_{1}}{2 \pi \lambda_{r} a_{00}} \frac{\left(\frac{\beta_{1} a_{00}}{\lambda_{0}}+a_{50}\right) a_{12} \lambda_{2}^{3}-a_{10} a_{52} \lambda_{0}^{3}}{a_{51} a_{12} \lambda_{2}^{3}-a_{52} a_{11} \lambda_{1}^{3}} \\
& A_{2}=\frac{Q \lambda_{2}}{2 \pi \lambda_{r} a_{00}} \frac{\left(\frac{\beta_{1} a_{00}}{\lambda_{0}}+a_{50}\right) a_{11} \lambda_{1}^{3}-a_{10} a_{51} \lambda_{0}^{3}}{a_{51} a_{12} \lambda_{2}^{3}-a_{52} a_{11} \lambda_{1}^{3}}
\end{aligned}
$$

for the half-space problem and

$$
A_{1}=-\frac{Q}{2 \pi a_{00} \sqrt{\lambda_{r} \lambda_{z}}} \frac{a_{12} \lambda_{2}^{2}-a_{10} \lambda_{0}^{2}}{a_{12} \lambda_{2}^{2}-a_{11} \lambda_{1}^{2}} \quad A_{2}=\frac{Q}{2 \pi a_{00} \sqrt{\lambda_{r} \lambda_{z}}} \frac{a_{11} \lambda_{1}^{2}-a_{10} \lambda_{0}^{2}}{a_{12} \lambda_{2}^{2}-a_{11} \lambda_{1}^{2}}
$$

for an infinite body.

The parameters $\lambda_{1}$ and $\lambda_{2}$ are the roots of the following equation

$$
c_{33} c_{44} \lambda^{4}-\left[c_{11} c_{33}-c_{13}\left(c_{13}+2 c_{44}\right)\right] \lambda^{2}+c_{11} c_{44}=0
$$

These parameters are the eigenvalues of the transversely isotropic material.

By defining

$$
\begin{aligned}
& \alpha=\sqrt{\frac{c_{11} c_{33}-c_{13}\left(2 c_{44}+c_{13}\right)+2 c_{44} \sqrt{c_{11} c_{33}}}{c_{33} c_{44}}} \\
& \beta=\sqrt{\frac{c_{11} c_{33}-c_{13}\left(2 c_{44}+c_{13}\right)-2 c_{44} \sqrt{c_{11} c_{33}}}{c_{33} c_{44}}}
\end{aligned}
$$


the eigenvalues $\lambda_{1}$ and $\lambda_{2}$ can be written as

$$
\lambda_{1}=\frac{1}{2}(\alpha+\beta) \quad \lambda_{2}=\frac{1}{2}(\alpha-\beta)
$$

It is noted that $\lambda_{1}$ and $\lambda_{2}$ can be either two positive real numbers or complex conjugate with a positive real part. In other words, $\alpha=\lambda_{1}+\lambda_{2}$ and it is always real. The results are valid even for the degenerate case of $\beta=\lambda_{1}-\lambda_{2}=0$, including the isotropic material where $\lambda_{1}=\lambda_{2}=1$. In this case, the limiting calculations with the use of de l'Hospital's rule give the solution.

\section{Conclusions}

- In comparison with the traditional methods applied to the solution of boundary value problems of thermoelasticity, in the proposed method, there is no need to solve boundary value problems of heat conduction for preliminary determination of the temperature field (the first stage of solving the problem) and then to solve the equations of thermoelasticity (the second stage of solving the problem).

- Green's functions for the half-space, infinite space made by multifield materials are obtained in an exact analytical form; the solutions are regular.

- For the temperature and heat flux applied along the circumference on an arbitrary plane, the thermal loading conditions may be written by means of the Dirac delta function. Then integration and/or superposition of Green's functions gives the multi-field result.

\section{Appendix A. The material coefficients for mulifield materials}

A1. The material parameters in characteristic equation (2.9) are as follows

$$
\begin{aligned}
& a=c_{44}\left[\mu_{33} e_{33}^{2}+\varepsilon_{33} q_{33}^{2}+c_{33} \mu_{33} \varepsilon_{33}-d_{33}\left(c_{33} d_{33}+2 e_{33} q_{33}\right)\right] \\
& b=\mu_{33}\left\{\left(e_{31}+e_{15}\right)\left[2 c_{13} e_{33}-c_{33}\left(e_{31}+e_{15}\right)\right]+2 c_{44} e_{33} e_{31}-c_{11} e_{33}^{2}-c_{33} c_{44} \varepsilon_{11}\right\} \\
& +\varepsilon_{33}\left\{\left(q_{31}+q_{15}\right)\left[2 c_{13} q_{33}-c_{33}\left(q_{31}+q_{15}\right)\right]+2 c_{44} q_{33} q_{31}-c_{11} q_{33}^{2}-c_{33} c_{44} \mu_{11}\right\} \\
& -\mu_{33} \varepsilon_{33} \widetilde{c}^{2}-\left(e_{31}+e_{15}\right)^{2} q_{33}^{2}-\left(q_{31}+q_{15}\right)^{2} e_{33}^{2}-c_{44} \mu_{11} e_{33}^{2}-c_{44} \varepsilon_{11} q_{33}^{2} \\
& +2 e_{33} q_{33}\left(q_{31}+q_{15}\right)\left(e_{31}+e_{15}\right)+d_{33}^{2} \widetilde{c}^{2}+2 c_{33} d_{33}\left(e_{31}+e_{15}\right)\left(q_{31}+q_{15}\right) \\
& +2 c_{44} c_{33} d_{11} d_{33}+2 e_{33} q_{33}\left(c_{44} d_{11}+c_{11} d_{33}\right)-2 d_{33}\left(c_{13}+c_{44}\right)\left[e_{33}\left(q_{31}+q_{15}\right)\right. \\
& \left.+q_{33}\left(e_{31}+e_{15}\right)\right] \\
& c=\mu_{33}\left\{2 e_{15}\left[c_{11} e_{33}-c_{13}\left(e_{31}+e_{15}\right)\right]+c_{44} e_{31}^{2}+\varepsilon_{11} \widetilde{c}^{2}\right\} \\
& +\varepsilon_{33}\left\{2 q_{15}\left[c_{11} q_{33}-c_{13}\left(q_{31}+q_{15}\right)\right]+c_{44} q_{31}^{2}+\mu_{11} \widetilde{c}^{2}\right\} \\
& +c_{33} c_{44} \mu_{11} \varepsilon_{11}+c_{11} c_{44} \mu_{33} \varepsilon_{33}+2\left(c_{13}+c_{44}\right)\left(q_{31}+q_{15}\right)\left(d_{11} e_{33}+d_{33} e_{15}-q_{33} \varepsilon_{11}\right) \\
& +2\left(c_{13}+c_{44}\right)\left(e_{31}+e_{15}\right)\left(d_{11} q_{33}+d_{33} q_{15}-e_{33} \mu_{11}\right) \\
& +\left(q_{31}+q_{15}\right)^{2}\left(c_{33} \varepsilon_{11}+2 e_{33} e_{15}\right)+\left(e_{31}+e_{15}\right)^{2}\left(c_{33} \mu_{11}+2 q_{33} q_{15}\right) \\
& -2\left(q_{31}+q_{15}\right)\left(e_{31}+e_{15}\right)\left(e_{33} q_{15}+q_{33} e_{15}+c_{33} d_{11}+c_{44} d_{33}\right) \\
& -2 c_{11} d_{33}\left(e_{33} q_{15}+q_{33} e_{15}\right)-2 c_{44} d_{11}\left(q_{33} e_{15}+e_{33} q_{15}\right) \\
& -2 c_{11} d_{11} q_{33} e_{33}-2 c_{44} d_{33} q_{15} e_{15}+2 c_{44} q_{15} q_{33} \varepsilon_{11} \\
& +2 c_{44} e_{15} e_{33} \mu_{11}+c_{11} q_{33}^{2} \varepsilon_{11}+c_{11} e_{33}^{2} \mu_{11}-2 \widetilde{c}^{2} d_{33} d_{11}-c_{11} c_{44} d_{33}^{2}-c_{44} c_{33} d_{11}^{2}
\end{aligned}
$$




$$
\begin{aligned}
d= & -c_{11} \mu_{33}\left(c_{44} \varepsilon_{11}+e_{15}^{2}\right)-c_{11} \varepsilon_{33}\left(c_{44} \mu_{11}+q_{15}^{2}\right)-c_{44}\left(e_{31}^{2} \mu_{11}+q_{31}^{2} \varepsilon_{11}\right)-e_{31}^{2} q_{15}^{2}-q_{31}^{2} e_{15}^{2} \\
& -\mu_{11} \varepsilon_{11} \widetilde{c}^{2}+d_{11} \widetilde{c}^{2}+2 c_{11} c_{44} d_{11} d_{33}+2 c_{13} q_{15} q_{31} \varepsilon_{11}+2 c_{13} e_{15} e_{31} \mu_{11}-2 c_{11} q_{15} q_{33} \varepsilon_{11} \\
& -2 c_{11} e_{15} e_{33} \mu_{11}+2 c_{13} q_{15}^{2} \varepsilon_{11}+2 c_{13} e_{15}^{2} \mu_{11}+2 e_{31} e_{15} q_{31} q_{15}+2 c_{11} e_{15} q_{15} d_{33} \\
& +d_{11}\left[-2 c_{13} e_{15}\left(q_{15}+q_{31}\right)-2 c_{13} q_{15}\left(e_{15}+e_{31}\right)\right]+d_{11}\left[2 c_{11}\left(e_{15} q_{33}+q_{15} e_{33}\right)+2 c_{44} e_{31} q_{31}\right] \\
e= & c_{11}\left[\mu_{11} e_{15}^{2}+\varepsilon_{11} q_{15}^{2}+c_{44} \varepsilon_{11} \mu_{11}-d_{11}\left(c_{44} d_{11}+2 e_{15} q_{15}\right)\right] \\
\widetilde{c}^{2}= & c_{11} c_{33}-c_{13}\left(c_{13}+2 c_{44}\right)
\end{aligned}
$$

A2. The parameters $a_{1}, b_{1}, c_{1}, d_{1}$, and $a_{2}, b_{2}, c_{2}, d_{2}$ in Eq. (2.8) are

$$
\begin{aligned}
& a_{1}=\beta_{1}\left[c_{33}\left(\varepsilon_{33} \mu_{33}-d_{33}^{2}\right)+\mu_{33} e_{33}^{2}+\varepsilon_{33} q_{33}^{2}-2 e_{33} d_{33} q_{33}\right]+\beta_{3}\left[-\left(c_{13}+c_{44}\right)\left(\varepsilon_{33} \mu_{33}-d_{33}^{2}\right)\right. \\
& \left.-\left(e_{31}+e_{15}\right)\left(\mu_{33} e_{33}-d_{33} q_{33}\right)-\left(q_{31}+q_{15}\right)\left(q_{33} \varepsilon_{33}-d_{33} e_{33}\right)\right] \\
& +\gamma_{3}\left[-\left(c_{13}+c_{44}\right)\left(d_{33} e_{33}-q_{33} \varepsilon_{33}\right)+\left(e_{31}+e_{15}\right)\left(d_{33} c_{33}+q_{33} e_{33}\right)\right. \\
& \left.-\left(q_{31}+q_{15}\right)\left(c_{33} \varepsilon_{33}+e_{33}^{2}\right)\right]+p_{3}\left[-\left(c_{13}+c_{44}\right)\left(d_{33} q_{33}-e_{33} \mu_{33}\right)\right. \\
& \left.+\left(q_{31}+q_{15}\right)\left(d_{33} c_{33}+q_{33} e_{33}\right)-\left(e_{31}+e_{15}\right)\left(c_{33} \mu_{33}+q_{33}^{2}\right)\right] \\
& b_{1}=\beta_{1}\left[c_{33}\left(2 d_{11} d_{33}-\varepsilon_{33} \mu_{11}-\mu_{33} \varepsilon_{11}\right)+c_{44}\left(d_{33}^{2}-\varepsilon_{33} \mu_{33}\right)-\varepsilon_{11} q_{33}^{2}-\mu_{11} e_{33}^{2}\right. \\
& \left.+2 d_{33}\left(e_{33} q_{15}+q_{33} e_{15}\right)+2 d_{11} e_{33} q_{33}-2 q_{15} q_{33} \varepsilon_{33}-2 e_{15} e_{33} \mu_{33}\right] \\
& +\beta_{3}\left[-\left(c_{13}+c_{44}\right)\left(2 d_{11} d_{33}-\varepsilon_{33} \mu_{11}-\mu_{33} \varepsilon_{11}\right)\right. \\
& +\left(q_{13}+q_{15}\right)\left(q_{15} \varepsilon_{33}+q_{33} \varepsilon_{15}-d_{11} e_{33}-d_{33} e_{15}\right) \\
& \left.+\left(e_{31}+e_{15}\right)\left(e_{15} \mu_{33}+e_{33} \mu_{11}-d_{11} q_{33}-d_{11} q_{15}\right)\right] \\
& +\gamma_{3}\left[\left(c_{13}+c_{44}\right)\left(d_{11} e_{33}+d_{33} e_{15}-q_{15} \varepsilon_{33}-q_{33} \varepsilon_{11}\right)\right. \\
& \left.-\left(e_{31}+e_{15}\right)\left(c_{44} d_{33}+c_{33} d_{11}+q_{15} e_{33}+e_{15} q_{33}\right)+\left(q_{31}+q_{15}\right)\left(c_{44} \varepsilon_{33}+c_{33} \varepsilon_{11}+2 e_{15} e_{33}\right)\right] \\
& +p_{3}\left[\left(c_{13}+c_{44}\right)\left(d_{11} q_{33}+d_{33} q_{15}-e_{15} \mu_{33}-e_{33} \mu_{11}\right)\right. \\
& \left.-\left(q_{31}+q_{15}\right)\left(c_{44} d_{33}+c_{33} d_{11}+q_{15} e_{33}+e_{15} q_{33}\right)+\left(e_{31}+e_{15}\right)\left(c_{44} \mu_{33}+c_{33} \mu_{11}+2 q_{15} q_{33}\right)\right] \\
& c_{1}=\beta_{1}\left[c_{44}\left(\varepsilon_{11} \mu_{33}+\varepsilon_{33} \mu_{11}-2 d_{11} d_{33}\right)+c_{33}\left(\varepsilon_{11} \mu_{11}-d_{11}^{2}\right)+\varepsilon_{33} q_{15}^{2}+\mu_{33} e_{15}^{2}\right. \\
& \left.-2 d_{11}\left(e_{15} q_{33}+q_{15} e_{33}\right)+2 q_{15} q_{33} \varepsilon_{11}+2 \mu_{11} e_{15} e_{33}\right] \\
& +\beta_{3}\left[\left(c_{13}+c_{44}\right)\left(d_{11}^{2}-\varepsilon_{11} \mu_{11}\right)-\left(e_{31}+e_{15}\right)\left(\mu_{11} e_{15}-d_{11} q_{15}\right)\right. \\
& \left.-\left(q_{31}+q_{15}\right)\left(\varepsilon_{11} q_{15}-d_{11} e_{15}\right)\right]+\gamma_{3}\left[\left(c_{13}+c_{44}\right)\left(q_{15} \varepsilon_{11}-e_{15} d_{11}\right)\right. \\
& \left.+\left(e_{31}+e_{15}\right)\left(d_{11} c_{44}+q_{15} e_{15}\right)-\left(q_{31}+q_{15}\right)\left(c_{44} \varepsilon_{11}+e_{15}^{2}\right)\right] \\
& +p_{3}\left[\left(c_{13}+c_{44}\right)\left(e_{15} \mu_{11}-q_{15} d_{11}\right)\right. \\
& \left.+\left(q_{31}+q_{15}\right)\left(d_{11} c_{44}+q_{15} e_{15}\right)-\left(e_{31}+e_{15}\right)\left(c_{44} \mu_{11}+q_{15}^{2}\right)\right] \\
& d_{1}=-\beta_{1}\left[c_{44}\left(\varepsilon_{11} \mu_{11}-d_{11}^{2}\right)+\mu_{11} e_{15}^{2}+\varepsilon_{11} q_{15}^{2}-2 e_{15} q_{15} d_{11}\right] \\
& a_{2}=c_{44}\left[\beta_{3}\left(\varepsilon_{33} \mu_{33}-d_{33}^{2}\right)+\gamma_{3}\left(d_{33} e_{33}+q_{33} \varepsilon_{33}\right)+p_{3}\left(d_{33} q_{33}-e_{33} \mu_{33}\right)\right] \\
& b_{2}=\beta_{1}\left[\left(c_{13}+c_{44}\right)\left(\varepsilon_{33} \mu_{33}-d_{33}^{2}\right)-\left(e_{31}+e_{15}\right)\left(d_{33} q_{33}-\mu_{33} e_{33}\right)\right. \\
& \left.-\left(q_{31}+q_{15}\right)\left(d_{33} e_{33}-\varepsilon_{33} q_{33}\right)\right]-\beta_{3}\left[c_{11}\left(\varepsilon_{33} \mu_{33}+d_{33}^{2}\right)\right. \\
& \left.+c_{44}\left(\mu_{11} \varepsilon_{33}+\mu_{33} \varepsilon_{11}\right)-2\left(q_{31}+q_{15}\right)\left(e_{31}+e_{15}\right) d_{33}+\left(q_{31}+q_{15}\right)^{2} \varepsilon_{33}+\left(e_{31}+e_{15}\right)^{2} \mu_{33}\right] \\
& -\gamma_{3}\left[c_{11}\left(d_{33} e_{33}-q_{33} \varepsilon_{33}\right)+c_{44}\left(d_{11} e_{33}+d_{33} e_{15}-q_{15} \varepsilon_{33}-q_{33} \varepsilon_{11}\right)\right. \\
& -\left(c_{13}+c_{44}\right) d_{33}\left(e_{31}+e_{15}\right)-q_{33}\left(e_{31}+e_{15}\right)^{2}+\varepsilon_{33}\left(q_{31}+q_{15}\right)\left(c_{13}+c_{44}\right) \\
& \left.+e_{33}\left(q_{31}+q_{15}\right)\left(e_{31}+e_{15}\right)\right]-p_{3}\left[c_{11}\left(d_{33} q_{33}-e_{33} \mu_{33}\right)\right. \\
& +c_{44}\left(d_{11} q_{33}+d_{33} q_{15}-e_{15} \mu_{33}-e_{33} \mu_{11}\right)-\left(c_{13}+c_{44}\right) d_{33}\left(q_{31}+q_{15}\right) \\
& \left.-e_{33}\left(q_{31}+q_{15}\right)^{2}+\mu_{33}\left(e_{31}+e_{15}\right)\left(c_{13}+c_{44}\right)+q_{33}\left(q_{31}+q_{15}\right)\left(e_{31}+e_{15}\right)\right]
\end{aligned}
$$




$$
\begin{aligned}
c_{2}= & \beta_{1}\left[-\left(c_{13}+c_{44}\right)\left(\varepsilon_{11} \mu_{33}+\varepsilon_{33} \mu_{11}\right)+\left(e_{31}+e_{15}\right)\left(d_{11} q_{33}+d_{33} q_{15}\right)\right. \\
& +\left(q_{31}+q_{15}\right)\left(d_{11} e_{33}+d_{33} e_{15}\right)-\left(q_{31}+q_{15}\right)\left(q_{15} \varepsilon_{33}+q_{33} \varepsilon_{11}\right) \\
& \left.-\left(e_{31}+e_{15}\right)\left(e_{15} \mu_{33}+e_{33} \mu_{11}\right)+2\left(c_{13}+c_{44}\right) d_{11} d_{33}\right]+\beta_{3}\left[c_{44}\left(\varepsilon_{11} \mu_{11}+d_{11}^{2}\right)\right. \\
& \left.+c_{11}\left(\mu_{11} \varepsilon_{33}+\mu_{33} \varepsilon_{11}\right)+\mu_{11}\left(e_{31}+e_{15}\right)^{2}+\varepsilon_{11}\left(q_{31}+q_{15}\right)^{2}-2\left(e_{31}+e_{15}\right)\left(q_{31}+q_{15}\right) d_{11}\right] \\
& -\gamma_{3}\left[c_{44}\left(q_{15} \varepsilon_{11}-e_{15} d_{11}\right)+\left(e_{31}+e_{15}\right)\left(\left(c_{13}+c_{44}\right) d_{11}+\left(e_{31}+e_{15}\right) q_{15}\right)\right. \\
& \left.-\left(q_{31}+q_{15}\right)\left(\left(c_{13}+c_{44}\right) \varepsilon_{11}+\left(e_{31}+e_{15}\right) e_{15}\right)-c_{11}\left(d_{11} e_{33}+e_{15} d_{33}-\varepsilon_{11} q_{33}-\varepsilon_{33} q_{15}\right)\right] \\
& -p_{3}\left[c_{44}\left(e_{15} \mu_{11}-q_{15} d_{11}\right)+\left(q_{31}+q_{15}\right)\left(\left(c_{13}+c_{44}\right) d_{11}+\left(q_{31}+q_{15}\right) e_{15}\right)\right. \\
& \left.-\left(e_{31}+e_{15}\right)\left(\left(c_{13}+c_{44}\right) \mu_{11}+\left(q_{31}+q_{15}\right) q_{15}\right)-c_{11}\left(d_{11} q_{33}+q_{15} d_{33}-\mu_{11} e_{33}-\mu_{33} e_{15}\right)\right] \\
d_{2}= & \beta_{1}\left[\left(c_{13}+c_{44}\right) \varepsilon_{11} \mu_{11}-\left(e_{31}+e_{15}\right) d_{11} q_{15}-\left(q_{31}+q_{15}\right) d_{11} e_{15}+\left(q_{31}+q_{15}\right) q_{15} \varepsilon_{11}\right. \\
& \left.+\left(e_{31}+e_{15}\right) \mu_{11} e_{15}-\left(c_{13}+c_{44}\right) d_{11}^{2}\right]-\beta_{3}\left[c_{11}\left(\varepsilon_{11} \mu_{11}-d_{11}^{2}\right)\right]-\gamma_{3}\left[c_{11}\left(e_{15} d_{11}-q_{15} \varepsilon_{11}\right)\right] \\
& -p_{3}\left[c_{11}\left(q_{15} d_{11}-e_{15} \mu_{11}\right)\right]
\end{aligned}
$$

A3. The parameters $a_{3 i}$ and $a_{4 i}$ in Eq. (2.8) are defined by the parameter $a_{1 i}$ as follows

$$
\begin{aligned}
& a_{3 i}=\left\{\left\{\beta_{1}\left[\left(e_{31}+e_{15}\right)\left(q_{33} \lambda_{i}^{2}-q_{15}\right)-\left(c_{13}+c_{44}\right)\left(d_{11}-d_{33} \lambda_{i}^{2}\right)\right]\right.\right. \\
& +\beta_{3}\left[\left(e_{31}+e_{15}\right)\left(q_{31}+q_{15}\right) \lambda_{i}+\left(c_{44} \lambda_{i}^{2}-c_{11}\right)\left(d_{11}-d_{33} \lambda_{i}^{2}\right)\right] \\
& \left.+p_{3}\left[\lambda_{i}\left(c_{13}+c_{44}\right)\left(q_{31}+q_{15}\right)+\left(c_{44} \lambda_{i}^{2}-c_{11}\right)\left(q_{33} \lambda_{i}^{2}-q_{15}\right)\right]\right\} a_{1 i} \lambda_{i} \\
& +\beta_{1}\left[\left(c_{33} \lambda_{i}^{2}-c_{44}\right)\left(d_{11}-d_{33} \lambda_{i}^{2}\right)-\left(q_{33} \lambda_{i}^{2}-q_{15}\right)\left(e_{33} \lambda_{i}^{2}-e_{15}\right)\right] \\
& +\beta_{3} \lambda_{i}\left[\left(c_{13}+c_{44}\right)\left(d_{11}-d_{33} \lambda_{i}^{2}\right)-\left(q_{31}+q_{15}\right)\left(e_{33} \lambda_{i}^{2}-e_{15}\right)\right] \\
& \left.+p_{3} \lambda_{i}\left[\left(c_{13}+c_{44}\right)\left(q_{33} \lambda_{i}^{2}-q_{15}\right)-\left(q_{31}+q_{15}\right)\left(c_{33} \lambda_{i}^{2}-c_{44}\right)\right]\right\} \\
& \text { - }\left\{p_{3} \lambda_{i}\left[\left(e_{31}+e_{15}\right)\left(q_{33} \lambda_{i}^{2}-q_{15}\right)-\left(q_{31}+q_{15}\right)\left(e_{33} \lambda_{i}^{2}-e_{15}\right)\right]\right. \\
& +\beta_{1}\left[\left(e_{33} \lambda_{i}^{2}-e_{15}\right)\left(d_{11}-d_{33} \lambda_{i}^{2}\right)-\left(q_{33} \lambda_{i}^{2}-q_{15}\right)\left(\varepsilon_{11}-\varepsilon_{33} \lambda_{i}^{2}\right)\right] \\
& \left.+\beta_{3} \lambda_{i}\left[\left(e_{31}+e_{15}\right)\left(d_{11}-d_{33} \lambda_{i}^{2}\right)-\left(q_{31}+q_{15}\right)\left(\varepsilon_{11}-\varepsilon_{33} \lambda_{i}^{2}\right)\right]\right\}^{-1} \\
& a_{4 i}=-\left\{\left\{\beta_{1}\left[\left(e_{31}+e_{15}\right)\left(e_{33} \lambda_{i}^{2}-e_{15}\right)-\left(c_{13}+c_{44}\right)\left(\varepsilon_{11}-\varepsilon_{33} \lambda_{i}^{2}\right)\right]\right.\right. \\
& +\beta_{3}\left[\left(e_{31}+e_{15}\right)^{2} \lambda_{i}+\left(c_{44} \lambda_{i}^{2}-c_{11}\right)\left(\varepsilon_{11}-\varepsilon_{33} \lambda_{i}^{2}\right)\right] \\
& \left.+p_{3}\left[\lambda_{i}\left(c_{13}+c_{44}\right)\left(e_{31}+e_{15}\right)+\left(c_{44} \lambda_{i}^{2}-c_{11}\right)\left(e_{33} \lambda_{i}^{2}-e_{15}\right)\right]\right\} a_{1 i} \lambda_{i} \\
& +\beta_{1}\left[\left(c_{33} \lambda_{i}^{2}-c_{44}\right)\left(\varepsilon_{11}-\varepsilon_{33} \lambda_{i}^{2}\right)-\left(e_{33} \lambda_{i}^{2}-e_{15}\right)^{2}\right] \\
& +\beta_{3} \lambda_{i}\left[\left(c_{13}+c_{44}\right)\left(\varepsilon_{11}-\varepsilon_{33} \lambda_{i}^{2}\right)-\left(e_{31}+e_{15}\right)\left(e_{33} \lambda_{i}^{2}-e_{15}\right)\right] \\
& \left.+p_{3} \lambda_{i}\left[\left(c_{13}+c_{44}\right)\left(e_{33} \lambda_{i}^{2}-e_{15}\right)-\left(e_{31}+e_{15}\right)\left(c_{33} \lambda_{i}^{2}-c_{44}\right)\right]\right\} \\
& \text {. }\left\{p_{3} \lambda_{i}\left[\left(e_{31}+e_{15}\right)\left(q_{33} \lambda_{i}^{2}-q_{15}\right)-\left(q_{31}+q_{15}\right)\left(e_{33} \lambda_{i}^{2}-e_{15}\right)\right]\right. \\
& +\beta_{1}\left[\left(e_{33} \lambda_{i}^{2}-e_{15}\right)\left(d_{11}-d_{33} \lambda_{i}^{2}\right)-\left(q_{33} \lambda_{i}^{2}-q_{15}\right)\left(\varepsilon_{11}-\varepsilon_{33} \lambda_{i}^{2}\right)\right] \\
& \left.+\beta_{3} \lambda_{i}\left[\left(e_{31}+e_{15}\right)\left(d_{11}-d_{33} \lambda_{i}^{2}\right)-\left(q_{31}+q_{15}\right)\left(\varepsilon_{11}-\varepsilon_{33} \lambda_{i}^{2}\right)\right]\right\}^{-1}
\end{aligned}
$$

A4. The roots of characteristic equation (2.9) are presented by the formulae (eigenvalues of multifield materials)

$$
\begin{aligned}
& \lambda_{1}^{2}=-\frac{b}{4 a}-\frac{1}{2} \sqrt{R_{5}+R_{6}}-\frac{1}{2} \sqrt{2 R_{5}-R_{6}+\frac{1}{4} \frac{R_{7}}{\sqrt{R_{5}+R_{6}}}} \\
& \lambda_{2}^{2}=-\frac{b}{4 a}-\frac{1}{2} \sqrt{R_{5}+R_{6}}+\frac{1}{2} \sqrt{2 R_{5}-R_{6}+\frac{1}{4} \frac{R_{7}}{\sqrt{R_{5}+R_{6}}}}
\end{aligned}
$$




$$
\begin{aligned}
& \lambda_{3}^{2}=-\frac{b}{4 a}+\frac{1}{2} \sqrt{R_{5}+R_{6}}-\frac{1}{2} \sqrt{2 R_{5}-R_{6}-\frac{1}{4} \frac{R_{7}}{\sqrt{R_{5}+R_{6}}}} \\
& \lambda_{4}^{2}=-\frac{b}{4 a}+\frac{1}{2} \sqrt{R_{5}+R_{6}}+\frac{1}{2} \sqrt{2 R_{5}-R_{6}-\frac{1}{4} \frac{R_{7}}{\sqrt{R_{5}+R_{6}}}}
\end{aligned}
$$

where

$$
\begin{array}{llrl}
R_{1} & =2 c^{3}-9 b c d+27 a d^{2}+27 b^{2} e-72 a c e & & R_{2}=c^{2}-3 b d+12 a e \\
R_{3}=\sqrt{R_{1}^{2}-4 R_{2}^{3}} & R_{4}=\sqrt[3]{\frac{1}{2}\left(R_{1}+R_{3}\right)} & \\
R_{5}=\frac{b^{2}}{4 a^{2}}-\frac{2 c}{3 a} & R_{6}=\frac{R_{2}}{3 a R_{4}}+\frac{R_{4}}{3 a} & R_{7}=\frac{b^{3}}{a^{3}}-\frac{4 b c}{a^{2}}+\frac{8 d}{a}
\end{array}
$$

\section{References}

1. Chen W.Q., LeE K.Y., Ding H.J., 2004, General solution for transversely isotropic magnetoelectro-thermo-elasticity and the potential theory method, International Journal of Engineering Science, 42, 1361-1379

2. Hou P.F., Leung A.Y.T., Ding H.J., 2008, A point heat source on the surface of a semi-infinite transversely isotropic electro-magneto-thermo-elastic material, International Journal of Engineering Science, 46, 273-285

3. Lekhnitskit S., 1963, Theory of Elasticity in an Anisotropic Elastic Body, Holden-Day Inc., San Francisco

4. Morse P.M., Feshbach H., 1953, Methods of Theoretical Physics, McGraw Hill, New York

5. Rogowski B., 2012, A concave indenter on a piezo-electro-magneto-elastic substrate or a layer elastically supported, The Journal of Strain Analysis for Engineering Design, 47, 6, 362-378

6. Rogowski B., 2013, Anti-plane crack emanating from the interface in a bounded smart PEMOelastic structure, International Journal of. Applied Mechanics and Engineering, 18, 4, 1165-1199

7. Rogowski B., 2014, The analysis of mode I conducting crack under general applied loads in piezoelectromagnetoelastic layer, International Journal of Engineering Science, 75, 11-30

8. Rogowski B., 2015, The transient analysis of conducting crack in magneto-electro-elastic halfspace under anti-plane mechanical and in-plane electric and magnetic impact, Archive of Applied Mechanics, 85, 29-50

9. Stroh A.H., 1958, Dislocations and cracks in anisotropic elasticity, Philosophical Magazine, 3, 625-646 